\title{
PERFORMANCES DE MÉMORISATION EN FONCTION DE LA MODALITÉ D'ENCODAGE AU COURS DES 24 HEURES CHEZ DES TRAVAILLEURS POSTÉS
}

E. Galy, J. F. Camps, C. Mélan

Presses Universitaires de France | « Le travail humain »

2004/1 Vol. 67 | pages 21 à 40

ISSN 0041-1868

ISBN 2130542891

Article disponible en ligne à l'adresse :

http://www.cairn.info/revue-le-travail-humain-2004-1-page-21.htm

\section{!Pour citer cet article :}

E. Galy et al., « Performances de mémorisation en fonction de la modalité d'encodage au cours des 24 heures chez des travailleurs postés », Le travail humain 2004/1 (Vol. 67), p. 21-40. DOI 10.3917/th.671.0021

Distribution électronique Cairn.info pour Presses Universitaires de France.

(C) Presses Universitaires de France. Tous droits réservés pour tous pays.

La reproduction ou représentation de cet article, notamment par photocopie, n'est autorisée que dans les limites des conditions générales d'utilisation du site ou, le cas échéant, des conditions générales de la licence souscrite par votre établissement. Toute autre reproduction ou représentation, en tout ou partie, sous quelque forme et de quelque manière que ce soit, est interdite sauf accord préalable et écrit de l'éditeur, en dehors des cas prévus par la législation en vigueur en France. Il est précisé que son stockage dans une base de données est également interdit. 


\title{
PERFORMANCES DE MÉMORISATION EN FONCTION DE LA MODALITÉ D'ENCODAGE AU COURS DES 24 HEURES CHEZ DES TRAVAILLEURS POSTÉS
}

\author{
par E. Galy ${ }^{\star}, J \cdot$ F. CAMPS ${ }^{\star \star}$ et C. MÉLAN*
}

\section{SUMMARY}

MEMORIZATION PERFORMANCE AS A FUNCTION OF ENCODING MODALITY ACROSS THE SHIFT WORKERS' 24-HOUR DAY

A serial probe recognition task was used to explore the effects of modality (visual vs. auditory) on memorization during the 24-hour day. The aim was to determine whether performance would vary with the level of vigilance, either in quantity (number of items remembered) or in quality (memorization strategies). In each of the six sessions run on different days at 4-hourly intervals (at 3, 7, 11, 1519 and 23 hours), volunteer shift workers first completed Thayer's (1989) questionnaire of subjective alertness and then performed the memory task. For each trial, six common nouns were presented one by one, followed by a probe that was or not issued from the list. The modality of word presentation varied randomly between encoding (list of words) and restitution (probe), allowing an investigation into intraand inter-modal word recognition to be carried out.

Overall recognition rates did vary according to modality at encoding, but not at restitution. Auditory list presentation resulted in a significant superiority in the morning when vigilance was lowest, thereby favouring the hypothesis of quantitative variations in memory performance during the 24-hour day. Even though overall recognition rates did not vary across test periods for either modality, a recency effect occurred at all delays following auditory list presentation, and in the afternoon following the visual list presentation. Variations in the shape of serial position curves across the 24-hour day were interpreted as changes in the strategies spontaneously adopted by subjects whilst completing the memory task.

The auditory superiority effect demonstrated in this field study is discussed in the light of the findings reported in laboratory studies, i.e. the chronological coding of auditory input, the persistence of the acoustic code and phonological recoding of visual input. Most importantly, the effect was robust and stable across the day, suggesting a number of potential applications, particularly during the night when shift workers' alertness is at its lowest.

Key words: Shift Work, Alertness, Presentation Modality, Auditory Superiority, Immediate Memory.

* Laboratoire Travail et Cognition, CNRS UMR 5551, Maison de la Recherche, Université Toulouse - Le Mirail, 5, allée Antonio-Machado, 31058 Toulouse Cedex 9. E-mail : egaly@univtlse2.fr

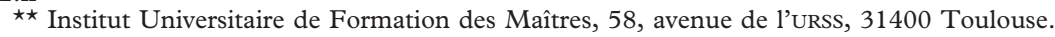

Le Travail Humain, tome 67, $\mathrm{n}^{\circ} 1 / 2004,21-40$ 


\section{INTRODUCTION}

L'automatisation et l'informatisation poussées des postes de travail au cours des 30 dernières années ont considérablement modifié la nature des tâches dévolues aux travailleurs postés, en particulier dans les secteurs industriels - énergie, chimie, sidérurgie - ainsi que dans les transports (Lejon, 1991). L'intervention directe et manuelle de l'homme s'est déplacée progressivement vers des activités mentales de commande et de coordination ; tâches de surveillance et de contrôle reposant sur le recueil et le traitement d'un nombre important d'informations. Parallèlement, le travail en équipes alternantes ou travail posté a connu une extension considérable (parmi les industries françaises dont la production est continue, $18 \%$ fonctionnent avec des équipes travaillant à horaires fixes contre $82 \%$ avec des équipes travaillant en horaires alternants). Or, la chronobiologie a apporté la preuve de l'existence de variations cycliques affectant le fonctionnement des différents systèmes biologiques des êtres vivants, l'homme y compris (Reinberg, 1979). La plupart des paramètres physiologiques et psychologiques de l'homme sont caractérisés par une alternance, au cours de la journée de 24 heures, entre des phases d'efficacité croissante et des phases d'efficacité réduite. Ces rythmes, dits circadiens, de loin les mieux connus, sont contrôlés en partie par un système endogène appelé circadien, lui-même sensible aux variations rythmiques qui se produisent dans l'environnement. Il s'agit d'une caractéristique fondamentale du fonctionnement humain constituant ce que Reinberg (1974) a appelé une "structure temporelle".

De nombreuses études portant sur les rythmicités de l'efficience cognitive se sont focalisées sur des tâches perceptives et motrices (recherche sérielle visuelle, temps de réaction, tri de cartes) qui requièrent un traitement immédiat d'informations. Elles ont montré, de manière typique, que les performances augmentent depuis de faibles niveaux le matin pour culminer en début de soirée avant de diminuer à nouveau, parallèlement au niveau de vigilance. De la même manière, des études réalisées auprès de travailleurs postés, au moyen d'enregistrements en continu de divers paramètres physiologiques (paramètres du rythme cardiaque, respiratoire, dosages hormonaux, température corporelle ; Monk \& Embrey, 1981 ; Rose, Jenkins, Hurst, Herd, \& Hall, 1982 ; Wotjczak-Jaroszowa \& Banaszkiewecz, 1974), de tests d'efficience cognitive (Folkard \& Monk, 1985 ; Maury \& Quéinnec, 1992 ; Tucker, Smith, Macdonald, \& Folkard, 1998) ainsi que d'analyses de l'activité de travail (Andorre \& Quéinnec, 1998), témoignent généralement d'un ralentissement nocturne des capacités fonctionnelles des travailleurs. Par conséquent, "l'accroissement de la charge de travail, induite par ces tâches (activités mentales de commande et de coordination), est d'autant plus sensible la nuit de par le bas niveau de vigilance de l'opérateur" (Poyet, 1990, p. 224). Les études de terrain révèlent, cependant, certaines distorsions par rapport au modèle sinusoïdal théorique de la rythmicité circadienne. Les conditions techniques, les contraintes des tâches et l'organisation du travail viennent médiatiser les rela- 
tions "état fonctionnel-efficience" de l'opérateur. Ainsi, la prise d'informations sur console, qui est globalement plus faible la nuit, est, par ailleurs, plus élevée en début de poste, indépendamment du poste (Andorre \& Quéinnec, 1996). De plus, d'autres facteurs qui viennent moduler le niveau de vigilance - fatigue, humeur, tension, anxiété, motivation, etc. - s'expriment différemment dans une étude de laboratoire que de terrain. C'est pourquoi, la compréhension des variations circadiennes des performances chez l'homme au travail est d'une importance évidente dans une perspective théorique, et pratique concernant l'organisation d'activités variées, notamment de travail.

Dans les salles de contrôle, la tâche de l'opérateur est plus centrée sur l'anticipation d'une situation critique que sur la détection de signaux d'alarmes. Dans ces situations de travail, la prise en compte et donc la mémorisation des états passés du système sont des facteurs essentiels (Marquié \& Quéinnec, 1987). L'évolution circadienne des performances dans des épreuves cognitives plus complexes que les tâches perceptivomotrices, et notamment mnésiques, reste néanmoins peu étudiée. Les travaux réalisés dans ce domaine ont révélé que les variations circadiennes des performances dans des tâches mnésiques dépendent de nombreux facteurs tels la charge mnésique, le délai de restitution, la profondeur de traitement, le type d'informations, l'épreuve de restitution (Folkard, Knauth, Monk, \& Rutenfranz, 1976 ; Folkard \& Monk, 1980 ; Lancry, 1986 ; Lorenzetti \& Natale, 1996 ; Maury \& Quéinnec, 1992 ; Oakhill \& Davies, 1989). Dans l'ensemble de ces études, et tel que le font remarquer Forêt et Benoît (1997, p. 35), "la quasi-totalité des épreuves de performance en laboratoire sont sur ordinateur et font intervenir les capacités visuelles du sujet. On doit donc être conscient qu'on a ainsi augmenté l'importance de cette modalité sensorielle, ce qui peut poser des problèmes quand on essaie de simuler des situations réelles ". Cette remarque prend tout son sens lorsque l'on prend en compte les données de la littérature qui montrent une supériorité des performances de mémorisation lors d'une présentation auditive d'un matériel verbal (Baddeley, 1993; Crowder \& Morton, 1969 ; Murray et al., 1999 ; Penney, 1989). Dans cette optique, nous avons étudié dans quelle mesure la modalité de présentation d'un matériel verbal influence la mémorisation de celui-ci compte tenu de l'état de vigilance des sujets. Nous nous sommes déplacés dans une entreprise de production d'énergie (centrale nucléaire) dont les opérateurs travaillent en horaires alternants à rotation rapide $(3 \times 8)$. La population étudiée est constituée d'agents de surveillance travaillant en équipes de quatre personnes. Ils se trouvent face à des écrans d'ordinateur sur lesquels ils peuvent afficher l'état des nombreux points de contrôle du site, et à des écrans de télévision reliés à des caméras de surveillance placées aux différents points d'accès de la centrale nucléaire. Dès qu'une anomalie se produit dans l'un des systèmes, les agents de surveillance consultent des cahiers de consignes relatives au type de problème rencontré. Ils sont donc amenés à garder en mémoire de travail les différentes consignes permettant de remédier à l'anomalie détectée. La démarche que nous avons choisie pour notre étude est une démarche expérimentale : les opérateurs ne sont pas confrontés à leur propre outil de travail qui sollicite diverses 
activités cognitives intimement imbriquées et rarement fixées se différenciant, en particulier, par le type de discrimination (simultanée ou successive, sensorielle ou cognitive), la modalité sensorielle (visuelle ou auditive) et la complexité de la source d'émission (simple ou multiple). Le matériel utilisé dans ce travail, correspondant à des listes de noms communs est, bien au contraire, simplifié afin de pouvoir contrôler les effets induits par la modalité. Cette étude est réalisée avec des travailleurs postés alors qu'ils sont en poste et cela à six moments différents de la journée. De ce point de vue, l'étude se déroule dans des conditions écologiques.

Dans la suite de cette introduction, nous résumerons les principales données de la littérature qui décrivent les fluctuations circadiennes de mémorisation chez l'homme, d'une part, et l'impact de la modalité de présentation de mots sur les performances de mémorisation, d'autre part.

\section{1. Fluctuations CiRCAdienNes de MÉmorisation CHEZ L'Adulte}

Un certain nombre de travaux se sont intéressés à la relation entre le niveau de vigilance et les performances de mémorisation. S'il semble actuellement bien établi que les performances humaines varient de manière systématique en fonction de l'heure du jour, de nombreuses questions restent ouvertes concernant la nature de ces changements et des mécanismes sous-jacents. Bien que des variations circadiennes des performances aient été systématiquement observées, celles-ci ne suivent pas le même décours temporel. Un problème urgent est de déterminer si les différences observées au cours de la journée représentent de réelles modifications de l'efficience. Il est possible que l'augmentation de l'éveil (arousal) circadien résulte d'une augmentation des ressources cognitives qu'un sujet peut utiliser dans une tâche. Dans ce cas, l'amélioration peut être décrite en termes de changements de la capacité effective. D'un autre côté, comme le suggèrent Monk et Lenk (1982) pour des tâches motrices, mais également de catégorisation de propositions logiques (Monk \& Carrier, 1997), il se pourrait que les effets observés soient dus au contraire à des changements de stratégie. Finalement, il est possible que des modifications à la fois de stratégies et de la capacité de traitement se produisent.

Les premiers travaux expérimentaux dans ce domaine ont montré que les rythmicités circadiennes de mémorisation varient en fonction du délai de rétention. Tel que le soulignent Folkard et Monk (1980), la capacité de traitement en MCT atteindrait son maximum en milieu de matinée et l'amélioration de la vigilance en cours d'après-midi améliore indirectement la rétention à long terme, indépendamment de l'heure de l'encodage. Folkard et al. (1976) et Lancry 1986 ont mis en avant que la charge mnésique joue un rôle essentiel dans l'évolution circadienne des performances. Les premiers montrent que les performances augmentent au cours de la journée, parallèlement au niveau de vigilance des sujets, dans une tâche de détection de deux lettres, alors que le profil inverse est observé lorsque la tâche porte sur six lettres. Lancry (1986) montre que dans des épreuves de rappel basées sur la catégorisation ou la reconnaissance, la charge mnémonique induite par l'encodage et le stockage 
d'informations serait trop faible pour saturer le système de traitement, tendant ainsi à masquer les rythmes circadiens du rappel. En d'autres termes, plus la charge mnésique d'une tâche est élevée, plus les fluctuations des performances selon le moment de la journée seraient prononcées. Ainsi, en fin d'après-midi, la capacité de traitement du sujet serait la plus grande, et donc moins rapidement saturée que le matin (Wright, Hull, \& Czeisler, 2002).

Plus récemment, Smith, McEvoy et Gevins (2002) montrent cependant que les performances en mémoire de travail diminuent significativement la nuit, à la fois lorsque la charge mnésique est faible ou élevée (comparer la position d'une lettre à celle respectivement de l'essai initial ou de deux essais plus tôt). De même, le rappel de quatre paires de mots seulement, à partir de la présentation d'un mot de chaque paire, est associé à une baisse significative des performances la nuit (Cajochen, Khalsa, Wyatt, Czeisler, \& Dijk, 1999 ; Wyatt, Cecco, Czeisler, \& Dijk, 1999).

Les résultats diffèrent sensiblement lorsque les tâches utilisées portent sur la compréhension et la mémorisation de matériels plus complexes, tels des textes et des phrases. Ces tâches impliquent différents processus cognitifs, mais il n'est pas clair si tous ces processus jouent un rôle dans les fluctuations circadiennes de performance. Folkard et Monk (1979 a; 1980) et Folkard et al. (1976) suggèrent que des changements des processus mnésiques et de compréhension au cours de la journée pourraient refléter des changements dans les stratégies que les sujets adoptent spontanément. La compréhension et le rappel de matériel linguistique présenté le matin semblent impliquer des processus de maintien de la forme superficielle de matériel linguistique, alors que du matériel présenté l'aprèsmidi est compris et mémorisé par des processus élaborés et intégrés. En outre, lorsque le type de stratégie utilisé est influencé en donnant comme consigne d'adopter une stratégie particulière pour réaliser les tâches, les fluctuations des performances observées habituellement dans des tâches de rappel immédiat peuvent être masquées (Folkard \& Monk, 1979 b). Dans les travaux de Oakhill (1988) et Oakhill et Davies (1989), les sujets lisent et mémorisent un texte à 9 heures ou à 17 heures et sont informés qu'ils passeraient ensuite une tâche de reconnaissance pour la moitié des sujets et une tâche de rappel pour l'autre moitié. Ces deux tâches favorisent respectivement le traitement des aspects de surface et de signification des items. Dans les deux cas, les auteurs observent une flexibilité des procédures adoptées, puisque le matin les sujets qui s'attendent à passer une épreuve de reconnaissance ont de meilleurs résultats que ceux qui s'attendent à passer une tâche de rappel. Ces données suggèrent alors que les variations d'efficience à des délais brefs traduisent des modifications qualitatives de traitement de l'information.

Selon Maury et Quéinnec (1993), ces résultats indiquent également que les modifications qualitatives de traitement peuvent résulter des processus d'encodage d'informations en mémoire à court terme. Pour tester cette hypothèse, ces auteurs étudient les variations d'efficience en mémoire, en fonction de la profondeur de traitement au moment de l'encodage qui dépendrait à son tour du niveau de vigilance des sujets. Les résultats indiquent qu'à 2 heures du matin, lorsque le profil de vigi- 
lance est faible, les sujets utiliseraient des procédures de traitement basées sur les caractéristiques physiques des stimuli, notamment visuelles et phonétiques, soutenues par des processus de répétition mentale. À l'inverse, des items traités à un niveau sémantique sont mieux mémorisés en fin d'après-midi que la nuit.

Par ailleurs, Maury et Quéinnec (1992) montrent que le temps de latence aux questions qui induisent les niveaux d'encodage est plus important pour les six derniers items de la liste, indépendamment du type de traitement induit. De la même manière, Folkard (1982) rapporte des variations circadiennes plus marquées pour les cinq ou six derniers items d'une liste. Ces données chronopsychologiques sont à rapprocher des études expérimentales portant sur la mémorisation de listes de mots. Ainsi, de nombreuses recherches (Craik \& Lockhard, 1972 ; Greene, 1987 ; Rundus, 1977 ; Wixted, 1991) tendent à montrer que les sujets débutent l'apprentissage d'une liste de mots en employant des processus de répétition élaborée, ceux-ci faisant place, en cours de liste, à des processus de répétition subvocale plus automatique pour les derniers items présentés.

\section{I.2. MODAlité PERCEPTIVE ET MÉMORISATION}

Le traitement des informations verbales à mémoriser entraîne de très nombreuses transformations, appelées codages. On peut ainsi parler de codes sensoriels (auditif, visuel), mais également de codes beaucoup plus élaborés, comme le code lexical ou le code sémantique. Chaque code a ses propres caractéristiques, et en particulier une durée de vie qui lui est propre. Ainsi, la mémoire d'une information peut être considérée comme un ensemble de toutes les informations codées (Lieury, 1992).

\section{I.2. A. Codage sensoriel}

Le stockage sensoriel visuel (iconique), mis en jeu lors d'une présentation visuelle de mots, persisterait de 200 à $400 \mathrm{~ms}$ après la disparition du stimulus (van der Heijden, 1981) et sa capacité serait d'environ 9 ou 10 items (Sperling, 1960). En revanche, le stockage sensoriel acoustique (échoïque), spécifique d'une présentation auditive des mots, peut durer 34 secondes et sa capacité est estimée à environ 5 items (Darwin, Turvey, \& Crowder, 1972).

Les deux registres sensoriels visuel et auditif présentent donc des différences de capacité et surtout de durée de stockage. Sur cette base, plusieurs études évaluant le rappel libre et/ou la reconnaissance de listes de mots ont montré l'existence d'un "effet de modalité " qui se traduit par une supériorité de restitution de mots ayant été présentés dans la modalité auditive. Murdock (1968) a montré, en premier, que la reconnaissance immédiate d'items verbaux est supérieure lorsque la présentation comporte une composante auditive, c'est-à-dire que le sujet entend les mots ou bien les lit à voix haute. Ce phénomène d'une supériorité auditive $\mathrm{a}$, par la suite, également été démontré lors de la restitution de mots en rappel libre ou indicé (Engle, 1974 ; Madigan, 1971 ; Murdock, 1967 ; Mur- 
dock \& Walker, 1969 ; Routh, 1976). Son effet s'observe généralement seulement au niveau des derniers mots d'une liste.

Une explication de ce phénomène basée exclusivement sur la persistance des codes sensoriels a été proposée par Crowder et Morton (1969), grâce à leur théorie du stockage acoustique précatégoriel (PAS). Selon celle-ci, la plus grande persistance du code échoïque par rapport au code iconique (visuel) expliquerait le rappel supérieur des derniers items d'une liste de mots entendus. Penney $(1975$; 1989) propose l'idée d'un traitement séparé des informations visuelles et auditives en mémoire à court terme grâce à des separate processing streams (flux de traitements séparés). Le code acoustique pourrait persister dans des conditions idéales jusqu'à 60 secondes, contre 1 à 2 secondes pour le code sensoriel visuel. Une activation nerveuse plus durable a, en effet, été démontrée lors d'un encodage auditif par rapport à un encodage visuel de mots (Rugg, Doyle, \& Mélan, 1993). Cette différence expliquerait le rappel supérieur des items présentés dans la modalité auditive ; supériorité qui pourrait alors concerner la totalité des mots d'une liste et non seulement les mots présentés en dernier.

Par ailleurs, le système sensoriel auditif traiterait les informations auditives de manière sérielle, ce qui favorise le traitement des aspects temporels et chronologiques des événements ainsi que la mémorisation de ces aspects. Par conséquent, lorsque des listes de mots sont à mémoriser, la chronologie de présentation des items pourrait contribuer à expliquer la supériorité auditive en mémoire à court terme (Murray et al., 1999 ; Penney, 1989). Le traitement sensoriel visuel en mode parallèle de mots présentés isolément favoriserait, en revanche, la mémorisation des aspects spatiaux et non sémantiques (Lehman, Mikesell, \& Doherty, 1985).

\section{I.2.B. Codage phonologique}

Le traitement des mots en mémoire à court terme implique ensuite un codage phonologique, lexical et sémantique. Le codage phonologique comporte deux composantes : la subvocalisation qui assure le recodage du graphique (visuel) en code phonologique lors de la lecture et l'autorépétition subvocale qui sert de mémoire "artificielle", permettant de prolonger la durée de vie de l'information phonologique (Lieury, 1992 ; Baddeley, 1993). En d'autres termes, les informations verbales visuelles subiraient un recodage phonologique alors que les informations auditives seraient automatiquement codées sous ce format, différence qui pourrait expliquer, en partie du moins, l'effet de modalité.

Parallèlement au codage phonologique, un codage lexical et sémantique s'opère, formats qui seraient moins nuancés selon la modalité d'encodage (Lieury, 1992 ; Ferrand, 2001).

Certains auteurs, s'intéressant à la fois à la mémorisation selon la modalité de présentation de mots et aux processus de compréhension de la parole et de la lecture, ont permis de proposer quelques recommandations pratiques. Ainsi, lors de la conception d'interfaces électroniques, Harvey (1999) propose d' "utiliser les messages linguistiques sonores 
pour les informations qui seront mises à profit rapidement et de privilégier le canal visuel pour les informations à mémoriser à plus long terme... Associer plusieurs systèmes de codes à un message important pour favoriser sa mémorisation... Demander à l'utilisateur de verbaliser les informations essentielles, peu importe leur mode de présentation... Utiliser la voix comme système d'entrée de données... Cette vocalisation facilite la mémorisation... Utiliser la narration pour les courtes listes d'éléments simples à mémoriser. Pour les listes plus longues ou les messages plus complexes, utiliser le texte..." (Harvey, 1999). Les quelques considérations indiquent alors que l'utilisation préférentielle de l'une ou l'autre modalité (visuelle ou auditive) ou format (liste, texte) dépend, en particulier de la quantité des informations à mémoriser, de leur importance et de la durée de mémorisation.

Dans ce travail, nous nous sommes attachés à un seul de ces facteurs, à savoir la modalité de présentation de mots dont nous avons exploré les effets dans une tâche de reconnaissance. Bien que certains auteurs aient décrit peu ou pas de variations avec ce type d'épreuves (Lancry, 1986), d'autres auteurs ont au contraire insisté sur leur utilité pour solliciter les processus de traitement de surface des mots (Oakhill, 1986 ; Lorenzetti \& Natale, 1996). De plus, l'épreuve de reconnaissance de mots permet une dissociation des effets de la modalité de présentation au moment de la phase d'encodage (liste de mots dans la modalité visuelle ou auditive) de ceux induits au moment de la phase de restitution (mot-test dans la modalité visuelle ou auditive). Le nombre de mots présentés à chaque essai est limité à six items, difficilement catégorisables, ce qui permet d'éviter des effets "plancher" et "plafond " dans ce type d'épreuve (Baddeley, Chincotta, Stafford, \& Turk, 2002). Ces précautions semblent particulièrement importantes lorsque l'on s'intéresse aux fluctuations des performances en fonction de la vigilance.

Par ailleurs, il existe actuellement un large consensus à travers les études qui évaluent la vigilance subjective des sujets parallèlement à leurs performances d'efficience cognitive, pour utiliser des échelles visuelles analogiques, des échelles graduées et des listes d'adjectifs (questionnaire de Thayer, 1986). Ces mesures de vigilance subjective sont à leur minimum vers cinq heures du matin, augmentent rapidement en début de matinée, puis plus lentement, pour être à leur maximum en cours d'aprèsmidi. La vigilance diminue à nouveau en fin de journée (Daurat, 1993 ; Galy, 2002 ; Owens et al., 2000 ; Smith et al., 2002 ; Thayer, 1967 ; Tirilly, 2002). Cette courbe est globalement similaire à celle que l'on obtient avec des indicateurs physiologiques de la vigilance, si ce n'est que le maximum est typiquement atteint plusieurs heures plus tôt.

Compte tenu de l'effet robuste de supériorité auditive observé systématiquement lors de la mémorisation à court terme de listes de mots, nous postulons que cet effet devrait se retrouver indépendamment du niveau de vigilance et concerner la partie récente de la liste (Baddeley, 1993 ; Crowder \& Morton, 1969) ou la totalité de la liste (Penney, 1989). Par ailleurs, si les sujets adoptent différentes stratégies de traitement selon leur niveau de vigilance, alors le processus de répétition mentale favoriserait la mémorisation des mots le matin. Cet effet devrait être plus pro- 
noncé pour une présentation auditive des mots subissant un recodage phonologique automatique. Selon l'hypothèse de capacité de traitement, on devrait observer, au contraire, de meilleures performances l'aprèsmidi, lorsque le niveau de vigilance est maximal. Le bénéfice observé serait alors plus marqué lors d'une présentation visuelle des mots. En effet, le codage étant moins direct et automatique dans cette modalité, on peut supposer que ces processus seraient favorisés par un niveau élevé de vigilance.

\section{MÉTHODE}

\section{1. SUJETS}

23 opérateurs volontaires d'une centrale de production d'énergie, travaillant en horaires alternants $(3 \times 8)$ en rotation rapide $(2$ matins, 2 aprèsmidis, 3 nuits, 5 jours de repos), ont participé à cette étude. Ils étaient âgés de 41,5 ans en moyenne (minimum 35 ans, maximum 52 ans) et pratiquaient des horaires alternants depuis 11,4 années en moyenne (minimum 4 ans, maximum 25 ans).

\section{2. MATÉRIEL}

\section{Épreuve mnésique}

Une tâche de reconnaissance sérielle (serial probe recognition task) était employée. 6 ensembles de 18 mots concrets, d'une même catégorie (animaux, légumes, etc..) étaient constitués en tenant compte de leur fréquence d'apparition dans le langage courant et du nombre de syllabes. Lors de chacun des 32 essais, les mots étaient présentés à la vitesse de 1 mot par seconde et, après un délai de rétention de quatre secondes, un mot-test était présenté. Simultanément, la consigne suivante apparaît " mot dans liste touche $\mathrm{Q}$, mot hors de la liste touche $\mathrm{M}$ ». La consigne restait affichée jusqu'à ce que le sujet réponde et le mot-test était issu de la liste présentée dans $50 \%$ des cas. Les mots de la série et le mot-test étaient présentés soit visuellement sur un écran d'ordinateur soit auditivement par un haut-parleur. De cette manière, quatre types d'essais ont pu être distingués : mots de la liste en auditif et mot-test en auditif (AA), auditif-visuel (AV), visuel-auditif (VA) ou visuel-visuel (VV).

Un logiciel permettait de faire varier le choix des mots, leur position dans la série et la modalité de présentation de manière pseudo-aléatoire d'essai en essai (Gellerman, 1933), avec les restrictions suivantes : tous les mots apparaissent un nombre équivalent de fois et une combinaison mot $\times$ position $\times$ modalité ne pouvait être répétée plus de deux fois consécutivement. L'épreuve durait environ douze minutes. Deux essais consécutifs étaient séparés par un délai de cinq secondes et un bip sonore signalait le début de l'essai suivant. 


\section{Évaluation de la vigilance}

À chaque point horaire, les sujets complétaient également l'échelle d'auto-évaluation de la vigilance de Thayer (1986). Celle-ci comporte 20 adjectifs ou expressions accompagnés chacun de 4 possibilités de réponse : "beaucoup, un peu, ne sais pas et pas du tout". La consigne précise d'éviter la réponse " je ne sais pas » et d'exécuter le test rapidement en consignant la première réponse qui vient à l'esprit. Chaque réponse est ensuite affectée d'une note de 1 à 4 . Ce questionnaire permet d'évaluer le niveau de vigilance du sujet (dimension $\mathrm{A}$ ) et sa tendance émotionnelle (dimension B). La dimension A, que nous avons utilisée, décrit le niveau d'activation générale (GA, évalué par les termes actif, énergique, dynamique, la pêche, en forme), d'une part, et la tendance au sommeil ou fatigue (DS, évaluée par endormi, somnolent, fatigué, attentif, éveillé), d'autre part. Le rapport GA/DS donne une évaluation subjective de la vigilance à un instant donné.

\section{II.3. ProcÉdure}

Les passations se déroulaient dans une salle isolée, à six points horaires différents du jour et de la nuit $(3 \mathrm{~h}, 7 \mathrm{~h}, 11 \mathrm{~h}, 15 \mathrm{~h}, 19 \mathrm{~h}, 23 \mathrm{~h})$. Afin d'éviter des effets liés à l'entraînement sur la tâche, les six points horaires étaient intégrés dans un plan d'expérience entièrement contrebalancé, avec un nombre identique de sujets commençant les tests à chacun des six horaires et ainsi de suite. De plus, deux passations ne pouvaient pas avoir lieu la même journée pour le même sujet. Chaque sujet a été familiarisé avec le matériel expérimental et avec la manipulation avant le début de l'expérience, en dehors des horaires d'observation.

Les sujets étaient installés face à un micro-ordinateur équipé d'un clavier et d'un haut-parleur. À chacun des 6 points horaires, ils complétaient le questionnaire de Thayer avant de réaliser l'épreuve mnésique. À la première passation (et si nécessaire aux suivantes), la procédure était expliquée au sujet à l'aide d'une nouvelle démonstration.

\section{4. StatistiQues}

Les performances de mémorisation des opérateurs sont évaluées par le pourcentage de mots reconnus 1) pour la totalité de la liste et 2) en fonction de la position du mot-test dans la liste. Puisque l'indépendance des données des 4 types d'essais n'est pas respectée, le recours à des statistiques non paramétriques était incontournable. C'est pourquoi la comparaison de ces données et l'analyse de l'évaluation au cours du nycthémère des indices de performance ainsi que l'indice de vigilance moyen ont été réalisées grâce à des tests non paramétriques pour échantillons appariés (test de Wilcoxon). De manière similaire, les analyses de corrélation des performances de mémorisation avec la vigilance subjective ont été effectuées à l'aide du test non paramétrique de Spearman. 


\section{RÉSULTATS}

\section{1. INDICE DE VIGILANCE}

Dans un premier temps, nous avons vérifié si les sujets présentaient bien un rythme de vigilance comparable à celui habituellement décrit (minimum en milieu de nuit et maximum en fin d'après-midi).

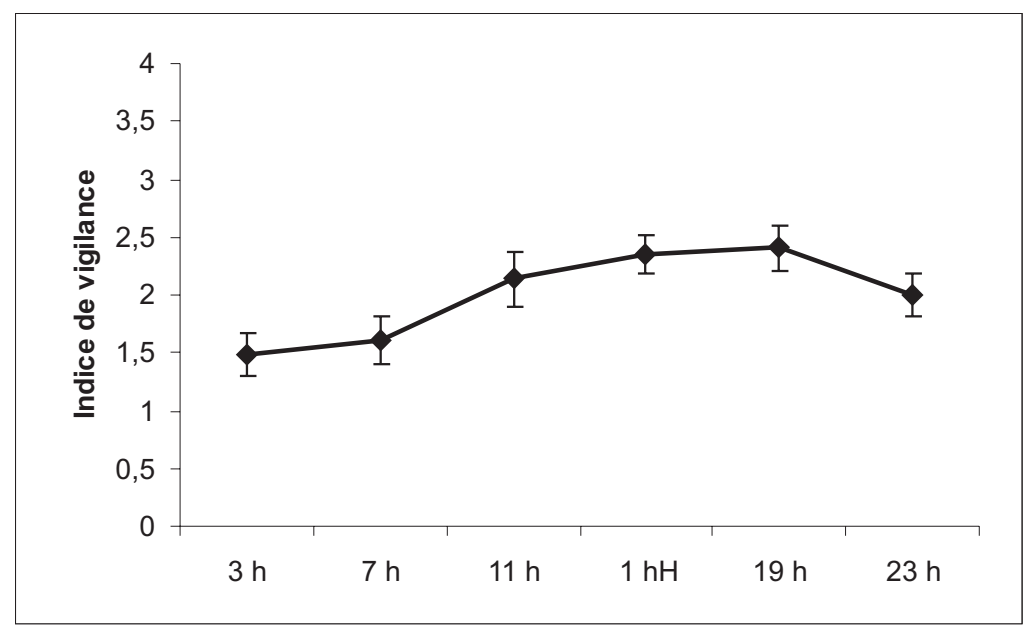

Fig. 1. - Indice moyen de vigilance (+/- erreur type) en fonction des horaires de passation

Index of alertness (+/- standard error) by Thayer's checklist as a function of time of day

La figure 1 montre que l'indice moyen de vigilance subjective varie entre 1,5 et 2,5 aux différents horaires de passation, sachant que la valeur maximale est de 4 . Le minimum observé se situe à $3 \mathrm{~h}$ du matin et le maximum à $19 \mathrm{~h}$. Les résultats statistiques montrent que la valeur de cet indice est significativement plus faible à $3 \mathrm{~h}$ qu'à $11 \mathrm{~h}, 15 \mathrm{~h}, 19 \mathrm{~h}$ et $23 \mathrm{~h}$ (respectivement $p<.005, p<.011, p<.003$, et $p<.050)$. Il existe également une différence significative entre $7 \mathrm{~h}$ et $15 \mathrm{~h}(7 \mathrm{~h}<15 \mathrm{~h} ; p<.022)$. Ces résultats indiquent alors une fluctuation du niveau de vigilance subjective au cours du nycthémère et, en particulier, une différence significative entre les horaires de jour et de nuit. L'évolution circadienne de la vigilance observée grâce au questionnaire de Thayer est similaire à celle décrite grâce à d'autres mesures subjectives et notamment, l'échelle visuelle analogique (Owens et al., 2000 ; Tirilly, 2002). Notons que le maximum apparaît relativement tôt, tout comme dans d'autres travaux utilisant des indices subjectifs de vigilance (Daurat et al., 1993 ; Tirilly, 2002). 


\section{2. PERFORMANCES DE MÉMORISATION}

\section{III.2.A. Performances globales de mémorisation} au cours du nycthémère en fonction de la modalité d'encodage

Dans ce paragraphe, nous avons analysé les pourcentages moyens de réponses correctes corrigées, correspondant à la fois aux mots de la liste correctement reconnus et à ceux n'ayant pas figuré dans la liste. L'analyse statistique des quatre types d'essais aux différents horaires ne révèle pas de différence significative selon la modalité de restitution. En effet, les conditions expérimentales AA et AV et les conditions VA et VV ne diffèrent pas entre elles et ce, à aucun des points horaires d'observation. Ce résultat nous a amené à regrouper les données en fonction de la modalité d'encodage (fig. 2).

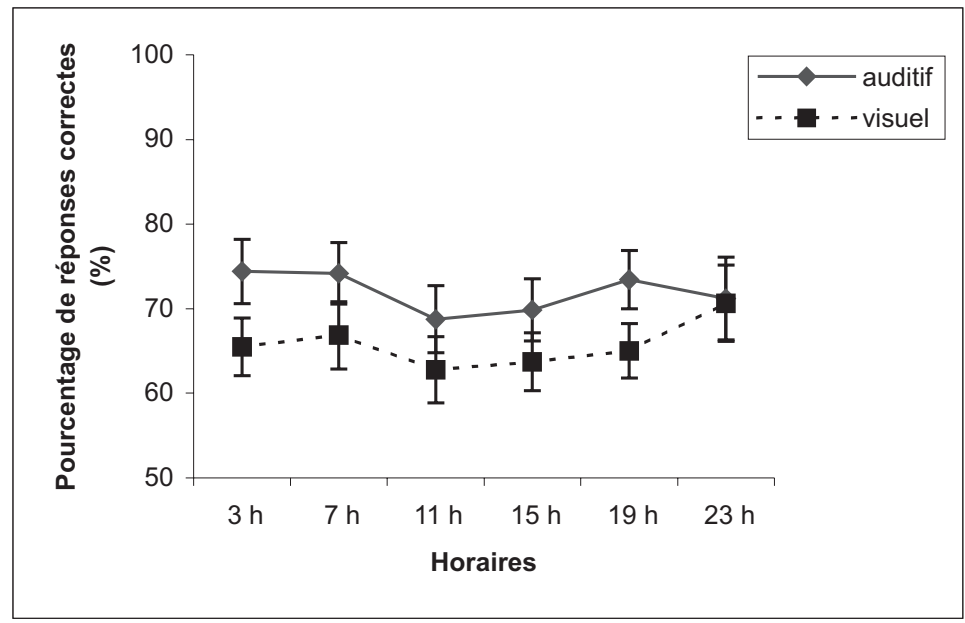

Fig. 2. - Pourcentages moyens de réponses correctes dans la liste et hors de la liste (+/- erreur type) en fonction de l'horaire lors d'une présentation auditive et visuelle des items

Mean percentage of correct responses to probes (+/- standard error) as a function of time of day following auditory and visual list presentation

La comparaison, à chaque point horaire, des performances de mémorisation des opérateurs en fonction de la modalité d'encodage indique que le pourcentage moyen de réponses correctes est supérieur suite à une présentation auditive de la liste de mots comparée à une présentation visuelle. Cette différence est significative à $3 \mathrm{~h}, 7 \mathrm{~h}$ et $11 \mathrm{~h}$ (respectivement, $p<.016, p<.037$ et $p<.009)$. En revanche, lorsque les performances sont comparées, séparément pour chaque modalité d'encodage, entre les différents horaires, aucune différence significative n'apparaît. 
Par ailleurs, lors d'une présentation visuelle des listes de mots, les performances sont positivement corrélées avec l'indice de vigilance $(r=.205 ; p<.025)$. En revanche, une relation similaire n'est pas observée lors d'une présentation auditive des listes.

Conformément à nos hypothèses, l'analyse des réponses correctes révèle une supériorité des performances lorsque les mots sont présentés dans la modalité auditive. Cette supériorité auditive peut être liée, d'une part, à un traitement en série des entrées auditives, susceptible de favoriser la mémorisation des aspects temporels et chronologiques des événements (Penney, 1989 ; Murray et al., 1999). En raison de cette caractéristique fonctionnelle, le canal auditif serait particulièrement adapté au traitement d'éléments sériels comme des listes de mots, en vue de leur mémorisation à court terme (Harvey, 1999 ; Morton, 1969 ; Penney, 1989 ; Watkins, Lecompte, Elliot, \& Fish, 1992). D’autre part, grâce au code acoustique, les informations auditives peuvent être récupérées sous cette forme pendant un laps de temps plus long que les informations visuelles, ce qui pourrait expliquer que le taux des réponses correctes est plus élevé dans ce cas (Crowder \& Morton, 1969 ; Penney, 1989). Selon cet auteur, le codage des aspects temporels des informations auditives se ferait dès le codage acoustique, ce qui rend les deux explications complémentaires. De plus, un recodage des entrées visuelles pourrait expliquer la mise en évidence de performances globales plus faibles dans cette modalité (Baddeley, 1993 ; Penney, 1989). L'analyse des courbes sérielles obtenues dans notre étude devrait révéler si la supériorité auditive est limitée à la portion finale de la courbe (Baddeley, 1993; Crowder \& Morton, 1969) ou si elle concerne la totalité de la courbe (Penney, 1989).

L'analyse des performances de mémorisation aux différents points horaires révèle que la supériorité auditive est significative seulement le matin ( $3 \mathrm{~h}, 7 \mathrm{~h}$ et $11 \mathrm{~h}$ ). Cela pourrait traduire la mise en jeu, par les sujets, d'un processus de répétition mentale qui serait particulièrement efficace lors du traitement des aspects phonologiques. Le recours à une stratégie de traitement des aspects de surface expliquerait alors la supériorité auditive lorsque la vigilance des sujets est faible (Folkard \& Monk, $1979 b$; Lorenzetti \& Natale, 1996). Néanmoins, étant donné la robustesse de ce phénomène, nous aurions dû l'observer également l'aprèsmidi. Nous pouvons alors penser que l'absence d'effet de modalité l'après-midi et le soir refléterait, en fait, une amélioration des performances de reconnaissance visuelle, plutôt qu'une disparition de la supériorité auditive. Ce résultat ainsi que la mise en évidence d'une corrélation entre le nombre de réponses correctes de mots présentés visuellement et la vigilance subjective des sujets sont en accord avec l'hypothèse de variations nycthémérales de la capacité de traitement. En effet, nous avions postulé que l'augmentation de la vigilance améliorerait le traitement plus "coûteux" (accès indirect au code phonologique) des informations visuelles. 
III.2.B. Performances de mémorisation au cours du nycthémère en fonction de la position des mots dans la liste et de la modalité d'encodage

Dans ce paragraphe, nous avons détaillé les résultats obtenus en fonction de la position que le mot-test occupait dans la liste, en excluant ainsi les résultats concernant les mots-tests hors liste. La figure 3 représente les courbes sérielles ainsi obtenues aux différents horaires d'observation.

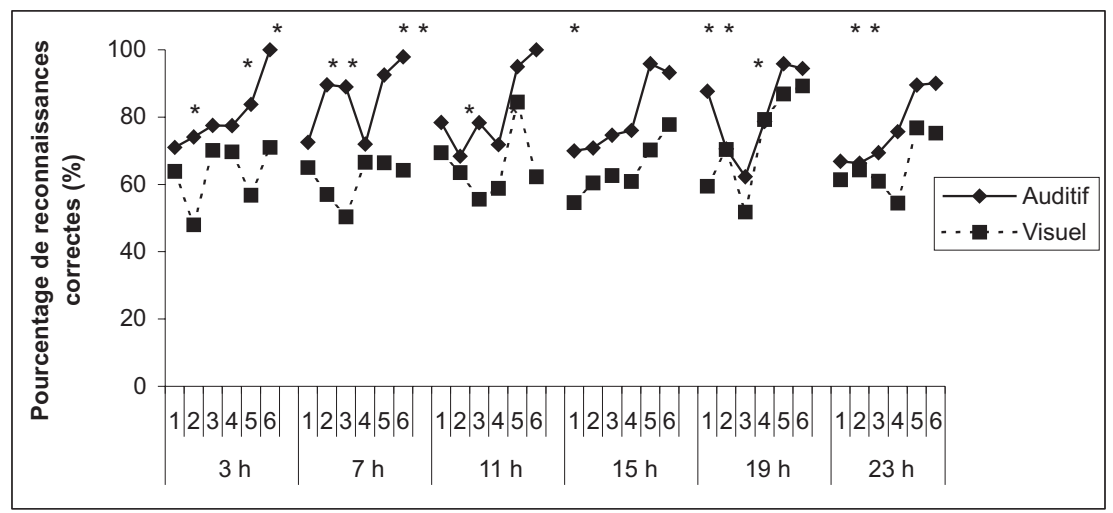

Fig. 3. - Pourcentage de reconnaissances correctes aux différents horaires en fonction de la position du mot dans la liste et de la modalité d'encodage ( ${ }^{\star}$ marque les points de la courbe pour lesquels il existe une différence significative entre une présentation auditive et visuelle)

Percentage of probes correctly recognized at different times of the day as a function of items' position of the initial list and modality of presentation ( ${ }^{\star}$ shows curve points where exists a significant difference between an auditory or visual presentation)

La comparaison des pourcentages de reconnaissances en fonction de la modalité de présentation révèle une supériorité auditive pour les deux derniers mots de la liste à $15 \mathrm{~h}, 19 \mathrm{~h}$ et $23 \mathrm{~h}(p<.015$ pour toutes les comparaisons) et pour pratiquement la totalité de la liste à $3 \mathrm{~h}, 7 \mathrm{~h}$ et $11 \mathrm{~h}$ (voir les détails sur la figure $3, p<.022$ pour toutes les comparaisons).

De plus, nous avons analysé les courbes sérielles, séparément pour chaque modalité, d'abord à chaque horaire, puis entre les différents horaires. Lors d'un encodage auditif des mots, un effet de récence significatif apparaît à tous les points horaires (supériorité des items en $5^{\mathrm{e}}$ et $6^{\mathrm{e}}$ position par rapport à ceux en $3^{\mathrm{e}}$ et $4^{\mathrm{e}}$ position, à $11 \mathrm{~h} p<.013$, à $15 \mathrm{~h}$ $p<.002$, à $19 \mathrm{~h} p<.001$, à $23 \mathrm{~h} p<.011$, à $3 \mathrm{~h} p<.012$ et à $7 \mathrm{~h}$ $p<.001)$. Cet effet de récence est stable tout au long des 24 heures. Un effet de primauté est également mis en évidence à 19 h (supériorité des 
items en $1^{\text {re }}$ et $2^{\mathrm{e}}$ position par rapport à ceux en $3^{\mathrm{e}}$ et $4^{\mathrm{e}}$ position, $p<.015$ ) et explique une meilleure reconnaissance des premiers items de la liste à $19 \mathrm{~h}$ par rapport à tous les autres points horaires $(p<.010$ pour toutes les comparaisons).

L'analyse des courbes sérielles, obtenues lors d'un encodage visuel, révèle également un effet de récence mais qui se manifeste seulement à $15 \mathrm{~h}$ et $19 \mathrm{~h}$ (respectivement, $p<.042$ et $p<.003$ ).

Ces résultats permettent d'affiner ceux obtenus au paragraphe précédent. Ils montrent, en particulier, que la différence des performances entre les deux modalités d'encodage concerne la portion initiale de la liste. En effet, la supériorité auditive est présente à tous les horaires pour toutes les portions de la liste, sauf l'après-midi au niveau de la portion initiale de la liste. Par ailleurs, lors d'un encodage visuel, un effet de récence apparaît l'après-midi. Prises ensemble, ces données appuient l'idée d'une amélioration du traitement des informations visuelles lorsque la vigilance subjective est maximale. Dans ce cas, l'absence d'effet de modalité l'après-midi, démontrée par l'analyse des performances globales, s'expliquerait par un traitement plus efficace en modalité visuelle. Par conséquent, si l'on estime que la supériorité des performances de reconnaissance des mots issus des positions initiales de la liste traduit effectivement un traitement moins efficace des informations visuelles (le matin), alors la véritable supériorité auditive serait restreinte à la portion récente de la liste. Ces données appuient alors l'hypothèse de Crowder et Morton (1969) proposant l'existence d'un code acoustique persistant pendant quelques secondes seulement. En revanche, ils ne sont pas en accord avec l'hypothèse de Penney (1989) selon laquelle l'effet de modalité aurait dû s'étendre sur la totalité de la liste, et cela indépendamment du niveau de vigilance des sujets.

Nos résultats ne permettent cependant pas de déterminer si l'effet de modalité s'opère au niveau du codage sensoriel (stock précatégoriel; Crowder \& Morton, 1969), et/ou au niveau du codage phonologique (Baddeley, 1993 ; Penney, 1989), voire à un niveau plus élaboré de traitement. Afin de répondre à cette question, des expériences complémentaires, actuellement en cours, visent à évaluer les effets du délai de rétention, de la longueur des listes de mots et du type d'épreuve de rappel (Galy, 2002). Ces études permettront également d'approfondir l'hypothèse des variations circadiennes qualitatives et/ou quantitatives en mémoire à court terme. Un délai de rétention plus court ou plus long devrait affecter plus particulièrement la portion récente de la courbe sérielle, alors qu'une augmentation du nombre de mots dans la liste permettrait d'éprouver l'hypothèse d'un traitement plus élaboré des informations de début de liste lorsque la vigilance est élevée.

De la même manière, il semble pertinent de faire varier les épreuves de restitution utilisées selon le type de stratégies de traitement explorées. Ainsi, les épreuves de reconnaissance se sont avérées utiles afin de montrer le recours à des stratégies de traitement des aspects de surface de mots ou de textes le matin (Lorenzetti \& Natale, 1996 ; Maury \& Quéinnec, 1992 ; Oakhill \& Davies, 1989). En revanche, les tests de rappel libre immédiat d'un texte seraient mieux adaptés pour évaluer le traitement 
d'informations intégrées et élaborées, plus performant l'après-midi (Folkard \& Monk, 1980 ; Lorenzetti \& Natale, 1996). De plus, des variations circadiennes des capacités de traitement des sujets peuvent être évaluées plus facilement avec des tâches de rappel qu'avec des tâches de reconnaissance (Lancry, 1986).

\section{DISCUSSION GÉNÉRALE}

Les résultats de cette étude confirment et étendent ceux obtenus par Maury et Quéinnec (1992), dans la mesure où les performances globales de mémorisation à court terme des opérateurs ne varient pas au cours du nycthémère, mais qu'une analyse détaillée fait apparaître des effets du format de présentation. De manière intéressante, nous avons mis en évidence que la reconnaissance de mots présentés visuellement varie avec le niveau de vigilance des sujets, alors qu'un tel effet est absent lors d'un encodage auditif. L'absence de fluctuations circadiennes globales des performances dans cette étude peut être attribuée à la charge mnémonique, relativement faible dans une tâche de reconnaissance, et ce d'autant plus que les listes de mots sont courtes (Lancry, 1986). Nous pouvons penser, en accord avec cet auteur, que la capacité de traitement du sujet ne serait pas saturée par ce type de tâche, ce qui masquerait alors d'éventuelles variations horaires des performances.

Les données rapportées dans cette étude sont, bien évidemment, valables pour certains types de messages et pour la population étudiée. Cependant, nous pouvons noter qu'elles appuient la proposition de Harvey selon laquelle la présentation auditive des informations devrait être utilisée lorsque ces informations doivent être mises à profit rapidement (Harvey, 1999). Ils suggèrent que cette proposition s'applique particulièrement bien aux énumérations, format que l'on trouve fréquemment utilisé pour les consignes de fonctionnement ou de sécurité. Notons que le canal auditif est multidirectionnel, et donc particulièrement utile pour attirer rapidement l'attention de l'opérateur et pour transmettre l'information urgente (Tucker \& Jones, 1991).

Compte tenu des caractéristiques du canal auditif, l'utilisation de messages sonores dans les salles de contrôle semble pertinente lorsque des informations en nombre limité doivent être traitées rapidement, et cela en particulier en période nocturne. Au contraire, les informations visuelles, comme nous l'avons montré, seraient traitées moins efficacement la nuit lorsque la vigilance est faible. Des analyses de l'activité de travail ont également montré que le nombre moyen de regards portés par des opérateurs en direction d'une zone informationnelle possible d'un écran de contrôle et la consultation de pages-écran est sensiblement plus faible la nuit que le matin et l'après-midi (Quéinnec \& de Terssac, 1980 ; Andorre \& Quéinnec, 1996). Rappelons que les informations, présentées par le canal visuel, ne pourront être appréhendées que lorsque les yeux balayent le champ adéquat et que l'opérateur y prête attention (Chatty, Athènes, \& Bustico, 1999). 
Il convient de noter toutefois le caractère éphémère des informations véhiculées par le canal auditif et le manque de contrôle du sujet sur le débit et l'enchaînement de l'information auditive. De plus, l'utilisation répétée ou inappropriée du canal sonore peut heurter l'intimité d'autrui de sorte que, dans certaines situations, ce dernier devient agaçant, voire nuisible. Par conséquent, afin d'améliorer la détection de signaux et leur traitement, il est nécessaire de considérer chaque poste de surveillance dans sa totalité et de manière indépendante et de tenter d'optimiser avec les intéressés, en particulier avec les opérateurs, le format de présentation des informations afin de contribuer à réduire la pénibilité du travail et de limiter les incidents liés à un niveau de vigilance diminué la nuit.

\section{BIBLIOGRAPHIE}

Andorre, V., \& Quéinnec, Y. (1996). La prise de poste en salle de contrôle de processus continu : approche chronopsychologique. Le Travail Humain, 59, 335354.

Andorre, V., \& Quéinnec, Y. (1998). Changes in supervisory activity of a continuous process during night and day shifts. Industrial Ergonomics, 21, 179-186.

Baddeley, A. D. (1993). La mémoire humaine : théorie et pratique. Grenoble : Presses Universitaires de Grenoble.

Baddeley, A. D. Chincotta, D., Stafford, L., \& Turk, D. (2002). Is the word length effect in STM entirely attributable to output delay ? Evidence from serial recognition. The Quarterly Fournal of Experimental Psychology, 55A, 353-369.

Cajochen, C., Khalsa, S. E. S., Wyatt, J. K., Czeisler, C. A., \& Dijk, D. J. (1999). EEG and ocular correlates of circadian melatonin phase and human performance decrements during sleep loss. American fournal of Physiology : Regulatory, Integrative and Comparative Physiology, 277, 640-649.

Chatty, S., Athènes, S., \& Bustico, A. (1999). Votre attention s'il vous plaît ! Éléments d'un espace de conception de signaux visuels. Proceedings de la $11^{e}$ conférence francophone de l'interaction homme-machine. Centre d'Études de la Navigation Aérienne, Toulouse.

Craik, F. I. M., \& Lockhart, R. S. (1972). Levels of processing : Framework for memory research. Fournal of Verbal Learning and Verbal Behavior, 11, 671-684.

Crowder, R. G., \& Morton, J. (1969). Precategorical acoustic storage (PAS). Perception and Psychophysics, 5, 365-373.

Darwin, C. J., Turvey, M. T., \& Crowder, R. G. (1972). An auditory analogue of the Sperling partial report procedure : Evidence for brief auditory storage. Cognitive Psychology, 3, 255-267.

Daurat, A., Aguirre, A., Foret, J., Bonnet, P., Keromes, A., \& Benoit, O. (1993). Bright light affects alertness and performance rhythms during a 24-h constant routine. Physiological Behavior, 53, 929-936.

Engle, R. W. (1974). The modality effect : Is precategorical acoustic storage responsible ? Fournal of Experimental Psychology, 102, 824-829.

Ferrand, L. (2001). Cognition et lecture : processus de base de la reconnaissance des mots écrits chez l'adulte. Bruxelles : DeBoëck Université.

Folkard, S. (1982). Circadian rhythms and human memory. In F. M. B. R. C. Gracher (Ed.), Rhythmic aspects of behaviour. Hillsdale, NJ : Lawrence Erlbaum Associates.

Folkard, S., Knauth, P., Monk, T. H., \& Rutenfranz, J. (1976). The effect of memory load on the corcadian variation in performance efficiency under a rapidly rotating shift system. Ergonomics, 19, 479-488. 
Folkard, S., \& Monk, T. H. (1979 a). Shift work and performance. Human Factors, 21, 483-492.

Folkard, S., \& Monk, T. H. (1979 b). Time of day and processing strategy in free recall. Quarterly fournal of Experimental Psychology, 31, 461-475.

Folkard, S., \& Monk, T. H. (1980). Circadian rhythms in human memory. British Fournal of Psychology, 71, 295-307.

Folkard, S., \& Monk, T. H. (1985). Circadian performance rhythms. In T. H. Monk \& S. Folkard (Eds.), Hours of Work Temporal Factors in Work Scheduling (pp. 37-52). Chichester, UK : John Wiley.

Forêt, J., \& Benoit, O. (1997). Rythmes circadiens et évolution circadienne de la vigilance. In D. L. C. Guilleminault (Éd.), Sommeil, vigilance et travail (pp. 3144). Paris: Masson.

Galy, E. (2002). Fluctuations circadiennes de performances de mémorisation et de discrimination chez des travailleurs postés : Rôle de la modalité perceptive et du niveau de vigilance. Thèse de doctorat, Université de Toulouse II.

Gellerman, L. W. (1933). Chance orders of alternative stimuli in visual discrimination experiments. Fournal of Genetic Psychology, 42, 206-208.

Greene, R. L. (1987). Effects of maintenance rehearsal on human memory. Psychological Bulletin, 102, 403-413.

Harvey, D. (1999). La multimédiatisation en éducation : vers une multimédiatisation efficace des messages dans les systèmes d'apprentissage multimédia interactif (SAMI). Paris : L'Harmattan.

Lancry, A. (1986). Mémoire et vigilance : approche chronopsychologique différentielle. Doctorat d'état, Université de Lille III.

Lehman, E. B., Mikesell, J. W., \& Doherty, S. C. (1985). Long-term retention of information about presentation modality by children and adults. Memory and Cognition, 13, 21-28.

Lejon, J.-C. (1991). L'évolution de la conduite sur SNCC. L'ergonomie des systèmes numériques de contrôle commande. Paris : ANACT.

Lieury, A. (1992). La mémoire : Résultats et théories. Liège : Mardaga.

Lorenzetti, R., \& Natale, V. (1996). Time of day and processing strategies in narrative comprehension. British fournal of Psychology, 87, 209-221.

Madigan, S. A. (1971). Modality and recall order interactions in short-term memory for serial order. Fournal of Experimental Psychology, 87, 294-296.

Marquié, J.-C., \& Quéinnec, Y. (1987). Vigilance et conditions de travail. Neuropsychologie, 2, 27-35.

Maury, P., \& Quéinnec, Y. (1992). Effects of 24-hour day on depth of processing in recall memory. British fournal of Psychology, 83, 249-260.

Maury, P., \& Quéinnec, Y. (1993). Qualitative changes in recall memory during day and night shifts. Ergonomics, 36, 289-297.

Monk, T. H., \& Carrier, J. (1997). Speed of mental processing in the middle of the night. Sleep, 20, 399-401.

Monk, T. H., \& Embrey, D. E. (1981). A field study of circadian rhythms in actual and interpolated task performance. In A. Reinberg, N. Vieux, \& P. Andlauer (Eds.), In Night and Shift Work: Biological and Social Aspects. Oxford : Pergamon Press.

Monk, T. H., \& Leng, V. (1982). Time of day effects in simple repetitive tasks : some possible mechanisms. Acta Psychologica, 51, 207-221.

Morton, J. (1969). Categories of interference : Verbal mediation and conflict in card sorting. British fournal of Psychology, 60, 329-346.

Murdock, B. B. (1967). Auditory and visual stores in short-term memory. Acta Psychologica, 27, 316-324.

Murdock, B. B. (1968). Modality effects in short-term memory : storage or retrieval ? Fournal of Experimental Psychology, 77, 79-86. 
Murdock, B. B., \& Walker, K. D. (1969). Modality effects in free recall. Fournal of Verbal Learning and Verbal Behavior, 8, 665-676.

Murray, D. J., Boudreau, N., Kurggraf, K. K., Dobell, L., Guger, S. L., Leask, A., Stanford, L., Tate, T. L., \& Wheeler, M. (1999). A grouping interpretation of the modality effect in immediate probed recognition. Memory and Cognition, 27, 234-245.

Oakhill, J. (1986). Effects of time of day on the integration of information in text. British Fournal of Psychology, 77, 481-488.

Oakhill, J. (1988). Text memory and integration at different times of day. Applied Cognitive Psychology, 2, 203-212.

Oakhill, J., \& Davies, A. M. (1989). The effects of time of day and subjects' test expectations on recall and recognition of prose materials. Acta Psychologica, $72,145-157$.

Owens, D. S., Macdonald, I., Tucker, P., \& Sytnik, N. (2000). Diurnal variations in the mood and performance of highly practised young women living under strictly controlled conditions. British Fournal of Psychology, 91, 41-60.

Penney, C. G. (1975). Modality effects in short-term verbal memory. Psychological Bulletin, 82, 68-84.

Penney, C. G. (1989). Modality effects and structure of short-term verbal memory. Memory and Cognition, 17, 398-422.

Poyet, C. (1990). L'homme, agent de fiabilité dans les systèmes automatisés. In G. de Terssac (Éd.), Les facteurs humains de la fiabilité dans les systèmes automatisés (pp. 223-241). Toulouse : Octarès.

Quéinnec, Y., \& de Terssac, G. (1980). Variation temporelle du comportement des opérateurs : le cas de processus à feu continu. Le Travail Humain, 44, 3953.

Reinberg, A. (1974). Chronopharmacology in man. Chronobiologia, 1, 157-191.

Reinberg, A. (1979). Le temps, une dimension biologique et médicale : chronobiologie et chronopathologie. In A. Reinberg, P. Fraisse, C. Leroy, H. Montagner, H. Pequignot, H. Poulizac, \& G. Vermeil (Eds.), L'homme malade du temps (pp. 24-62). Paris : Stock.

Rose, R. M., Jenkins, D., Hurst, M., Herds, J. A., \& Hall, R. P. (1982). Endocrine activity in air traffic controllers at work. II. Biological, psychological and work correlates. Psychoneuroendocrinology, 7, 113-123.

Routh, D. A. (1976). An "across-the-board" modality effect in immediate serial recall. Quarterly fournal of Experimental Psychology, 28, 285-304.

Rugg, M. D., Doyle, M. C., \& Mélan, C. (1993). An event-related potential study of the effects of intra- and inter-modal word repetition. Language and Cognitive Processes, 8, 357-377.

Rundus, D. (1977). Maintenance rehearsal and single-level processing. Fournal of Verbal Learning and Verbal Behavior, 16, 665-681.

Smith, M. E., McEvoy, L. K., \& Gevins, A. (2002). The import of moderate sleep loss on neurophysiologic signals during working-memory task performance. Sleep, 25, 784-794.

Sperling, G. (1960). The information available in brief visual representations. Psychological Monographs, 74, 1-29.

Thayer, R. E. (1967). Measurement of activation through self-report. Psychological Report, 20, 663-678.

Thayer, R. E. (1986). Activation-Deactivation adjective check-list : current overview and structural analysis. Psychological Report, 58, 607-614.

Tirilly, G. (2002). Ajustement du rythme veille-sommeil et de la vigilance aux horaires fractionnés en mer : influence des facteurs circadiens et sociaux. Thèse de doctorat, Université de Toulouse II.

Tucker, P., \& Jones, D. M. (1991). Voice as interface : an overview. International fournal of Human-Computer Interaction, 3, 145-169. 
Tucker, P., Smith, L., Macdonald, I., \& Folkard, S. (1998). Shift length as a determinant of retrospective on-shift alertness. Scand $\mathcal{F}$ Work Environ Health, 24 (suppl. 3), 49-53.

Van der Heijden, A. H. C. (1981). Short-term Visual Information Forgetting. London : Routledge \& Kegan Paul.

Watkins, M. J., LeCompte, D. C., Elliot, M. N., \& Fish, B. S. (1992). Short-term memory for the timing of auditory and visual signals. Fournal of Experimental Psychology, 18, 931-937.

Wixted, J. T. (1991). Conditions and consequences of maintenance rehearsal. Fournal of Experimental Psychology : Learning, Memory and Cognition, 17, 963973.

Wojtczak-Jaroszowa, J., \& Banaszkiewecz, A. (1974). Physical work capacity during the day and at night. Ergonomics, 17, 193-198.

Wright, K. P., Hull, J. T., \& Czeisler, C. A. (2002). Relationship between alertness, performance, and body temperature in humans. American fournal of Physiology: Regulatory, Integrative and Comparative Physiology, 283, 1370-1377.

Wyatt, J. K., Ritz-De Cecco, A., Czeisler, C. A., \& Dijk, D. J. (1999). Circadian temperature and melatonin rhythms, sleep, and neurobehavioral function in humans living on a 20-h day. American fournal of Physiology : Regulatory, Integrative and Comparative Physiology, 277, 1152-1153.

RÉSUMÉ

Les effets de la modalité de présentation (auditive vs visuelle) sur la mémorisation sont explorés chez des travailleurs postés à 3, 7, 11, 15, 19 et 23 heures dans une tâche de reconnaissance sérielle. Une supériorité d'un encodage auditif des mots est mise en évidence tout au long du nycthémère et expliquée par les particularités fonctionnelles de ce canal perceptif et $d u$ traitement des informations auditives visuelles en mémoire à court terme. Lors d'une présentation visuelle des mêmes mots, l'allure des courbes sérielles de mémorisation fluctue parallèlement au niveau de vigilance des opérateurs. En l'absence de différence du nombre total de mots reconnus lorsqu'ils sont présentés auditivement, ces résultats plaident en faveur de variations circadiennes des stratégies de traitement que les opérateurs adopteraient spontanément dans ce type de tâche. L'absence de fluctuations circadiennes des performances de reconnaissance lors d'un encodage auditif des mots, suggère des applications potentielles de ce format de présentation pour certains types de messages et cela, en particulier la nuit, lorsque le niveau de vigilance diminue.

Mots-clés: Travail posté, Vigilance, Modalité d'encodage, Supériorité auditive, Mémoire à court terme.

Manuscrit reçu : octobre 2002. Accepté par J.-M. Hoc après révision : octobre 2003. 